\title{
Breast cancer hypofractionated radiotherapy in 2-weeks with 2D technique: 5 -year clinical outcomes of a phase 2 trial
}

\author{
Budhi Singh Yadav ${ }^{1}$, Divya Dahiya ${ }^{2}$, Ankita Gupta ${ }^{3}$, Diksha Rana ${ }^{1}$, Ngangom Robert ${ }^{1}$, Manorma Sharma ${ }^{1}$, \\ Bharatbhushan $\mathrm{Rao}^{3}$
}

\author{
${ }^{1}$ Radiation Oncology, Postgraduate Institute of Medical Education and Research, Chandigarh, India \\ ${ }^{2}$ Department of General Surgery, Postgraduate Institute of Medical Education and Research, Chandigarh, India \\ ${ }^{3}$ Department of Radiotherapy, Postgraduate Institute of Medical Education and Research, Chandigarh, India
}

\begin{abstract}
Background: To report clinical outcomes and late toxicities of a 2-week hypofractionated post-operative loco-regional radiotherapy in patients with breast cancer.

Materials and methods: This trial was approved by the Institutional Ethics Committee and registered with gov, no. NCT02460744. Between June 2013 and October 2014, 50 patients with breast cancer, post mastectomy or breast conserving surgery (BCS) were included in this study, of whom 10 had BCS. Patients were planned on a 2-dimentional (2D) simulator with 2 tangential fields and an incident supraclavicular field. Radiotherapy dose was $34 \mathrm{~Gy} / 10 \# / 2$ weeks and a sequential boost of $10 \mathrm{~Gy} / 5 \# / 1$ wk in BCS patients. The primary endpoint was the rate of acute skin toxicities previously reported. Here, we report the secondary end points of late toxicities, cosmesis, local recurrence, disease-free survival (DFS) and overall survival (OS). Late skin toxicities were recorded according to the Radiotherapy and Oncology Group (RTOG) scoring criteria. Cosmetic outcomes were assessed using the Harvard/National Surgical Adjuvant Breast and Bowel Project (NSABP)/RTOG breast cosmesis and the Late Effects Normal Tissue/Subjective Objective Management Analytic (LENT/SOMA) scales for the breast and chest wall, respectively. Kaplan-Meier estimates of DFS and OS were calculated, and 5-year DFS and OS rates (with approximate 95\% Cls) were estimated.

Results: Late grade $\geq 2$ chest wall induration, hypopigmentation and subcutaneous fibrosis were seen in $3(6 \%), 3(6 \%)$ and $1(2 \%)$ patients, respectively. Chest wall cosmesis was excellent/good in 34 (72\%) and fair/bad in $13(28 \%)$ patients. In BCS patients, grade 2 skin induration, subcutaneous fibrosis and edema was observed in 1 patient (11\%) each. Cosmesis was excellent/good in 7 (78\%) and fair/bad in 2 (22\%) patients. Late grade $\geq 2$ arm edema, pain and shoulder stiffness were reported by $1(2 \%), 2(4 \%)$ and $2(4 \%)$ patients, respectively. No local recurrences were observed. Five patients developed distant metastases (10\%). Seven patients died (14\%). The 5-year DFS and OS rate was 90\% (95\% Cl: $77-96 \%)$ and $88 \%$ (95\% Cl: 75-94\%), respectively.

Conclusion: Hypofractionated radiotherapy in 2 weeks in patients with breast cancer was associated with minimal late toxicity, good cosmetic outcome and excellent local control. This trial may be of relevance for developing countries where resources are limited.
\end{abstract}

Key words: breast cancer; hypofractionated radiotherapy; post-mastectomy; late effects

Rep Pract Oncol Radiother 2021;26(4):503-511

Address for correspondence: Dr Budhi Singh Yadav, MD, Additional Professor, Radiation Oncology, PGIMER, Sector-12, Chandigarh, fax: +91-172-2744401 (W), tel: +91-172-2756390 (W), e-mail: drbudhi@gmail.com

This article is available in open access under Creative Common Attribution-Non-Commercial-No Derivatives 4.0 International (CC BY-NC-ND 4.0) license, allowing to download articles and share them with others as long as they credit the authors and the publisher, but without permission to change them in any way or use them commercially 


\section{Introduction}

Radiotherapy is an essential component of breast cancer management [1]. Hypofractionation is gaining momentum in breast cancer [2-4]. Studies are going on to reduce total treatment duration by delivering a larger dose per fraction in these patients. In spite of continuous research, the limit for hypofractionation is not yet reached. Taking a step in this direction, we have previously reported feasibility and early toxicity results from a phase 2 study of 2-week post-operative loco-regional radiotherapy for patients with breast cancer [5]. Hypofractionated radiation therapy within 2 weeks appeared to be feasible and was associated with an acute toxicity profile that was similar to what has been reported with a 3-week treatment. Preliminary estimates of 3 -year disease-free survival (DFS) and overall survival (OS) rates were $94 \%$ and $96 \%$, respectively, after a median follow-up of 39 months.

In this manuscript, we report results for late skin toxicities, updated 5-year late toxicity rates, and clinical outcomes after a median follow-up of 60 months.

\section{Materials and methods}

This phase 2 trial was approved by the Institutional Ethics Committee and registered with ClinicalTrials.gov, no. NCT02460744. Patient and tumor characteristics, as well as details of the treatment regimen, have been previously described [1]. Briefly, between June 2013 and October 2014, 50 patients with breast cancer, post mastectomy or after breast conserving surgery (BCS) were included in this phase 2 study, of whom 10 had BCS. Inclusion criteria were; age $\geq 18$ years, female or male patients with invasive carcinoma of the breast, BCS or mastectomy, $\geq 10$ axillary lymph nodes dissected, complete microscopic excision of primary tumour, T1-4 pN0-3 M0 disease, neoadjuvant or adjuvant chemotherapy, written informed consent and able to comply with follow up. None of the patients received implants or reconstruction. Exclusion criteria were; past history of malignancy, except (i) basal cell skin cancer and CIN cervix uteri or (ii) non-breast malignancy allowed if treated with curative intent and at least 5-year disease free and contralateral breast cancer, including ductal carcinoma in-situ (DCIS), irrespective of date of diagnosis.

\section{Radiotherapy}

Patients were planned on 2-dimensional (2D) fluoroscopic conventional simulator in a supine position on a breast board with ipsilateral arm abducted to $90^{\circ}$. Treatment was done with two tangential opposing fields to the breast/chest wall and a single incident field to the supraclavicular fossa. Field marking for the breast/chest wall included the midline, medially; the midaxillary fold, laterally, the 2nd intercostal space, cranially, and $1 \mathrm{~cm}$ below the opposite inframammary fold, caudally. For the supraclavicular field (SCF), the caudal border was the cranial border of breast/chest wall field, cranially, the thyroid notch, medially, along the medial border of the sternocleidomastoid muscle and, laterally, the insertion of deltoid. There was no gap between the tangential and SCF borders.

Central lung distance (CLD) was kept between 1-2.5 cm. Radiotherapy dose delivered was 34 Gy/10\#/2 weeks prescribed at mid separation. SCF dose was prescribed at dmax on cobalt or $6 \mathrm{MV}$ linac. Internal mammary nodes were not treated in this study. No cardiac shielding was done. BCS patients were treated on $6 \mathrm{MV}$ linac and a $30^{\circ}$ wedge was also used. A boost of $10 \mathrm{~Gy} / 5 \# / 1 \mathrm{wk}$ was given to cases with BCS with photons or electrons. Boost was planned with digital reconstructed radiographs on computerized tomography (CT).

Mastectomy patients were treated on cobalt machine with a breast cone, if separation was $>20 \mathrm{~cm}$ then they were also treated on linac. Chest wall bolus was used for $50 \%$ of treatment days (for 5 fractions).

\section{Dosimetry}

Dosimetry was done in 20 patients with left breast cancer. Patients were planned on a 2D simulator on a breast board. Field borders were set and CLD was calculated. Field borders were marked. After that, patients were taken to a CT-simulator for 3-dimensional (3D) treatment planning. The patients were positioned supine on the same breast board with the same parameters in the same position. Lead wires were placed on the field borders and $100 \mathrm{ml}$ of intravenous contrast was given. CT axial cuts were taken from the level of the larynx to the upper abdomen, including both lungs with a scan thickness and index of $3 \mathrm{~mm}$. CT images were transferred to the treatment planning system (TPS). The chest wall, heart, bilateral lungs, 
left anterior descending coronary artery (LAD) and opposite breast were contoured. The treatment parameters, patients and organ at risk outlines were exported to TPS. 3D planning was used to reconstruct $2 \mathrm{D}$ radiotherapy target volume for treating the chest and loco regional lymph nodes. Plans were made using standard tangential fields. Heart, bilateral lungs, LAD artery and opposite breast dose volume histograms were generated. From these, mean doses to the heart, LAD, proximal LAD, distal LAD, bilateral lung and opposite breast, V5 of the right lung, V5, V10 and V20 of the left lung and V2 of the opposite breast were calculated.

\section{Assessments}

Patients were examined by radiation oncologists every 3 months for the first year, every 6 months till 5 years for tumour recurrence and late toxicities. BCS cosmetic outcomes were assessed by a clinician using the Harvard/National Surgical Adjuvant Breast and Bowel Project (NSABP)/Radiotherapy and Oncology Group (RTOG) breast cosmesis grading scale before treatment and then on regular follow up. Chest wall cosmesis and late skin toxicities were reported as per RTOG radiation morbidity scoring criteria [1]. Any symptoms for pulmonary fibrosis, rib fractures and cardiac problems were also noted.

\section{Outcomes}

The primary endpoint was the rate of acute skin toxicities which has been previously published. Acute grade 2 and 3 skin toxicity was seen in 16 $(32 \%)$ and $1(2 \%)$ patients, respectively [1]. Here, we report results for the secondary endpoint of physician reported late toxicities and updated results for the secondary endpoints of cosmetic outcome, local recurrence, DFS and OS. Late skin toxicities were defined as any toxicity occurring after 6 months of radiotherapy. DFS was defined as time from treatment to recurrence or metastasis. OS was defined from the date of diagnosis till death due to breast cancer or last follow up date.

\section{Statistical analyses}

The primary objective was to obtain estimates of rates of acute toxicities and cosmetic outcomes that could be used to design a subsequent phase 3 comparative study. The present study included 50 patients. A sample size of 50 patients would provide a $95 \%$ confidence interval (CI) that would be less than $\pm 10 \%$ of any estimated rate. Our BCS rat is $25-30 \%$ of all patients, so 10 patients with BCS were included in this study, but the numbers are inadequate to separately analyze and distinguish BCS from mastectomy patients. The primary endpoint was the rate of acute skin toxicities. Secondary endpoints included cosmetic outcome, late toxicity, local recurrence, disease-free survival (DFS) and overall survival (OS). For cosmesis, the results were dichotomized as the proportion of patients with an excellent or good result versus the proportion with fair or poor result. Length of follow-up was calculated as the time from recruitment until the time of the first event or last follow-up assessment, whichever occurred first. Kaplan-Meier estimates of DFS and OS were calculated, and 5-year relapse and mortality rates, (with approximate 95\% CIs) were estimated. All statistical analyses were performed using SPSS (Statistical Package for the Social Sciences) v.16.0.

\section{Results}

Mean age was $51 \pm 12$ years. Left-sided tumors were in $25(50 \%)$ patients. Total mastectomy with axillary clearance (TMAC) was done in $40(80 \%)$ and BCS in 10 (20\%) patients (Tab. 1). Histopathology was luminal A, luminal $\mathrm{B}$, Her2/neu rich and TNBC in 12 (24\%), 8 (16\%), 10 (20\%) and 20 (40\%) patients, respectively. Regional nodal irradiation was done in all patients. Median tangential chest wall/breast separation was $18 \mathrm{~cm}$ (range 15 to $25 \mathrm{~cm}$ ). CLD was $2 \mathrm{~cm}$ (range 1.4 to $2.8 \mathrm{~cm}$ ).

Median follow-up was 60 months (range 14-100 months). Late toxicities were assessed in 47 of the 50 patients (Tab. 2). Three patients died, 2 because of infection and 1 due to metastatic disease before late toxicity assessment. Physician reported late grade $\geq 2$ chest wall induration, hypopigmentation and subcutaneous fibrosis at 5 years were seen in 3 (6\%), 3 (6\%) and 1 (2\%) patients, respectively. Late toxicities did not deteriorate over the years. Chest wall edema subsided with time. It was seen in $2(4 \%)$ patients at 3 years. At 5 years, chest wall edema was not observed in any patient.

Chest wall cosmesis was excellent/good in 34 patients (72\%, 95\% CI: 57-84\%) and fair/bad in 13 patients $(28 \%, 95 \%$ CI: $16-43)$. Only 1 patient $(2 \%)$ 
Table 1. Patient and tumor characteristics

\begin{tabular}{|c|c|c|}
\hline Characteristic & $n(\%)$ & $95 \% \mathrm{Cl}$ \\
\hline Mean age(range) & $51(26-75)$ & \\
\hline \multicolumn{3}{|l|}{ Comorbidity } \\
\hline Hypertension & $9(18)$ & $7-34$ \\
\hline Diabetes & $4(8)$ & $2-22$ \\
\hline None & $37(74)$ & $57-87$ \\
\hline \multicolumn{3}{|l|}{ Laterality } \\
\hline Right & $25(50)$ & $33-67$ \\
\hline Left & $25(50)$ & $33-67$ \\
\hline \multicolumn{3}{|l|}{ Histology } \\
\hline \multicolumn{3}{|l|}{ T-stage (clinical) } \\
\hline $\mathrm{T} 1 \& 2$ & $33(66)$ & $49-81$ \\
\hline $\mathrm{T} 3 \& 4$ & $17(34)$ & $19-51$ \\
\hline \multicolumn{3}{|l|}{ N stage } \\
\hline pNO & $23(46)$ & $30-63$ \\
\hline pN1a & $21(42)$ & $26-59$ \\
\hline pN2a & $5(10)$ & $3-24$ \\
\hline pN3a & $1(2)$ & $0-13$ \\
\hline \multicolumn{3}{|l|}{ Grade } \\
\hline 2 & $27(54)$ & $37-70$ \\
\hline 3 & $23(46)$ & $30-63$ \\
\hline \multicolumn{3}{|l|}{ Ki-67 } \\
\hline$>35 \%$ & $9(18)$ & $7-34$ \\
\hline$\leq 35 \%$ & $41(82)$ & $66-93$ \\
\hline \multicolumn{3}{|l|}{ Surgery } \\
\hline Mastectomy & $40(80)$ & $64-91$ \\
\hline Breast conserving & $10(20)$ & $9-36$ \\
\hline \multicolumn{3}{|l|}{ Surgical margins } \\
\hline Negative & $47(94)$ & $81-99$ \\
\hline Positive & $3(6)$ & $1-19$ \\
\hline \multicolumn{3}{|l|}{ Oestrogen receptor } \\
\hline Positive & $25(50)$ & $33-67$ \\
\hline Negative & $25(50)$ & $33-67$ \\
\hline \multicolumn{3}{|l|}{ Progesterone receptor } \\
\hline Positive & $20(40)$ & $24-57$ \\
\hline Negative & $30(60)$ & $43-76$ \\
\hline \multicolumn{3}{|l|}{ HER 2} \\
\hline Positive & $10(20)$ & $9-36$ \\
\hline Negative & $40(80)$ & $64-91$ \\
\hline \multicolumn{3}{|l|}{ Chemotherapy } \\
\hline Yes & $47(94)$ & $81-99$ \\
\hline Neoadjuvant & $22(44)$ & $28-61$ \\
\hline Adjuvant & $25(50)$ & $33-67$ \\
\hline No & $3(6)$ & $1-19$ \\
\hline \multicolumn{3}{|l|}{ Hormone therapy } \\
\hline Yes & $25(50)$ & $33-67$ \\
\hline No & $25(50)$ & $33-67$ \\
\hline \multicolumn{3}{|l|}{ Trastuzumab } \\
\hline Yes & $1(2)$ & $0-13$ \\
\hline No & $9(18)$ & $7-34$ \\
\hline
\end{tabular}

95\% confidence intervals $(\mathrm{Cl})$ based on exact binomial distributions had grade 3 late toxicity in the form of induration. There was no detrimental effect on chest wall cosmesis over the years.

Nine of the 10 patients with BCS were assessed for late toxicities; one died due to metastatic disease. Grade 2 skin induration, subcutaneous fibrosis and edema were observed in 1 patient (11\%) each. Cosmesis was excellent/good in 7 patients (78\%, 95\% CI: 40-97\%) and fair/bad in 2 patients (22\%, 95\% CI 3-60\%). Cosmesis was also stable over time. There was no grade 3 late breast toxicity. There was no telangiectasia.

Arm and shoulder function were assessed in 46 patients (Tab. 3). Four patients could not be assessed for arm and shoulder function. Late grade $\geq 2$ arm edema, pain and shoulder stiffness were reported by 1 patient ( $2 \%, 95 \%$ CI: $0-12 \%)$, 2 patients (4\%, 95\% CI: $1-15 \%)$ and 2 patients (4\%, 95\% CI: 1-15\%), respectively. Arm and shoulder functions did not deteriorate with time. There was no brachial plexopathy, rib fracture, pulmonary or cardiac toxicity. No second cancers have been reported.

No local recurrences were observed. Five patients developed distant metastases (10\%, 95\% CI: 1.2-16.5\%): 2 lung, 1 bone, 1 liver and 1 ascites. Among these, luminal A, Her2/neu rich and TNBC were found in 1 (2\%), $2(4 \%)$ and $2(4 \%)$ patients, respectively. Seven patients died $(14 \%, 95 \% \mathrm{CI}$ : 5-28\%); 5 due to metastases (10\%, 95\% CI 3-24\%) and 2 because of infection (4\%, 95\% CI: $0-13 \%)$. The 5-year DFS rate was $90 \%$ (95\% CI: 77-96\%) (Fig. 1) and the 5 -year OS rate was $88 \%$ (95\% CI: 75-94\%) (Fig. 2).

Estimated Dmax was up to $120 \%$ of the prescribed dose and the prescribed dose coverage of the target was $92-95 \%$ of the prescribed dose. Mean doses to the heart, LAD, proximal LAD and distal LAD were $3.07 \pm 1.25$ Gy $(\mathrm{EQD} 2=2.03 \mathrm{~Gy}), 12.92 \pm 6.46 \mathrm{~Gy}$ $(\mathrm{EQD} 2=11.09 \mathrm{~Gy}), 3.60 \pm 3.81 \mathrm{~Gy}(\mathrm{EQD} 2=2.42$ Gy) and 21.14 \pm 9.77 Gy (EQD2 = 21.62 Gy), respectively (Fig. 3). Mean doses to the left lung, V5, V10 and V20 were 8.24 \pm 3.50 Gy (EQD2 = 6.30 Gy), $28.40 \pm 13.56 \%, 24.59 \pm 10.61 \%$ and $20.00 \pm 9.15 \%$, respectively (Fig. 4). Mean dose to opposite breast was $0.43 \pm 0.31$ Gy (EQD2 = 0.26 Gy). Brachial plexus was contoured in 15 patients. Brachial plexus Dmean and Dmax were $29.54 \pm 11.25$ Gy (EQD2 = $35.18 \mathrm{~Gy})$ and $37.63 \pm 16.63 \mathrm{~Gy}$ $($ EQD2 $=50.90$ Gy), respectively (Fig. 5). Dmax to 
Table 2. Late toxicities

\begin{tabular}{|c|c|c|c|c|c|c|c|c|}
\hline \multirow{3}{*}{ Late toxicity } & \multicolumn{4}{|c|}{ All patients } & \multicolumn{4}{|c|}{ Patients with BCS } \\
\hline & \multicolumn{2}{|c|}{3 year $(n=48)$} & \multicolumn{2}{|c|}{5 year $(n=47)$} & \multicolumn{2}{|c|}{3 year $(n=10)$} & \multicolumn{2}{|c|}{5 year $(n=9)$} \\
\hline & $\mathrm{n}(\%)$ & $95 \% \mathrm{Cl}$ & n (\%) & $95 \% \mathrm{Cl}$ & n (\%) & $95 \% \mathrm{Cl}$ & n (\%) & $95 \% \mathrm{Cl}$ \\
\hline \multicolumn{9}{|l|}{ Skin induration } \\
\hline RTOG Grade 0 & $28(58)$ & $43-72$ & $28(60)$ & $44-74$ & $4(40)$ & $12-74$ & $4(44)$ & $14-79$ \\
\hline RTOG Grade 1 & $17(35)$ & $22-51$ & $16(34)$ & $21-49$ & $4(40)$ & $12-74$ & $4(44)$ & $14-79$ \\
\hline RTOG Grade 2 & $2(4)$ & $1-14$ & $2(4)$ & $1-15$ & $2(20)$ & $3-56$ & $1(11)$ & $0-48$ \\
\hline RTOG Grade 3 & $1(2)$ & $0-11$ & $1(2)$ & $0-11$ & $0(0)$ & $0-31$ & $0(0)$ & $0-34$ \\
\hline \multicolumn{9}{|c|}{ Hypopigmentation } \\
\hline RTOG Grade 0 & $46(96)$ & $86-99$ & $44(94)$ & $82-99$ & $10(100)$ & $69-100$ & $9(100)$ & $66-100$ \\
\hline RTOG Grade 2 & $2(4)$ & $1-14$ & $3(6)$ & $1-18$ & $0(0)$ & $0-31$ & $0(0)$ & $0-34$ \\
\hline \multicolumn{9}{|c|}{ Subcutaneous fibrosis } \\
\hline RTOG Grade 0 & $47(98)$ & $89-100$ & $46(98)$ & $89-100$ & $9(90)$ & $56-100$ & $8(89)$ & $52-100$ \\
\hline RTOG Grade 2 & $1(2)$ & $0-11$ & $1(2)$ & $0-11$ & $1(10)$ & $0-44$ & $1(11)$ & $0-48$ \\
\hline \multicolumn{9}{|c|}{ Chest wall edema } \\
\hline RTOG Grade 0 & $46(96)$ & $86-99$ & $47(100)$ & $92-100$ & $8(80)$ & $44-97$ & $8(89)$ & $52-100$ \\
\hline RTOG Grade 2 & $2(4)$ & $1-14$ & $0(0)$ & $0-8$ & $2(20)$ & $3-56$ & $1(11)$ & $0-48$ \\
\hline \multicolumn{9}{|l|}{ Cosmesis } \\
\hline Excellent/Good & $34(71)$ & $56-83$ & $34(72)$ & $57-84$ & $8(80)$ & $44-97$ & $7(78)$ & $40-97$ \\
\hline Fair/Poor & $14(29)$ & $17-44$ & $13(28)$ & $16-43$ & $2(20)$ & $3-56$ & $2(22)$ & $3-60$ \\
\hline
\end{tabular}

BCS - breast conserving surgery; RTOG — Radiation Therapy Oncology Group Cl - confidence interval

Table 3. Arm and shoulder function

\begin{tabular}{|c|c|c|c|c|}
\hline \multirow{2}{*}{ Toxicity } & \multicolumn{2}{|c|}{3 year $(n=48)$} & \multicolumn{2}{|c|}{5 year $(n=46)$} \\
\hline & $\mathrm{n}(\%)$ & $95 \% \mathrm{Cl}$ & $\mathrm{n}(\%)$ & $95 \% \mathrm{Cl}$ \\
\hline \multicolumn{5}{|l|}{ Arm edema } \\
\hline RTOG Grade 0 & $4390)$ & $77-97$ & $41(89)$ & $76-96$ \\
\hline RTOG Grade 1 & $4(8)$ & $2-20$ & $4(9)$ & $2-21$ \\
\hline RTOG Grade 2 & $0(0)$ & $0-7$ & $0(0)$ & $0-8$ \\
\hline RTOG Grade 3 & $1(2)$ & $0-11$ & $1(2)$ & $0-12$ \\
\hline \multicolumn{5}{|l|}{ Arm pain } \\
\hline RTOG Grade 0 & $46(96)$ & $86-99$ & $44(96)$ & 85-99 \\
\hline RTOG Grade 1 & $2(4)$ & $1-14$ & 2(4) & $1-15$ \\
\hline \multicolumn{5}{|c|}{ Shoulder stiffness } \\
\hline RTOG Grade 0 & $47(98)$ & $89-100$ & $44(96)$ & $85-99$ \\
\hline RTOG Grade 2 & $1(2)$ & $0-11$ & $2(4)$ & $1-15$ \\
\hline
\end{tabular}

RTOG — Radiation Therapy Oncology Group Cl — confidence interval; Cl — confidence interval

the brachial plexus was consistent between $35.30 \mathrm{~Gy}$ $(\mathrm{EQD} 2=46.10 \mathrm{~Gy})$ to $39.25 \mathrm{~Gy}(\mathrm{EQD} 2=54.36 \mathrm{~Gy})$.

\section{Discussion}

In this phase 2 study of 2-week hypofractionation in patients with breast cancer with a $2 \mathrm{D}$ tech- nique, late toxicities were acceptable, cosmesis was good and local control was excellent. Overall adverse events were low. Late grade 3 skin toxicity was observed only in $1(2 \%)$ patient in the form of skin induration of the chest wall.

The objective of loco-regional irradiation in breast cancer is to reduce loco-regional recurrence 


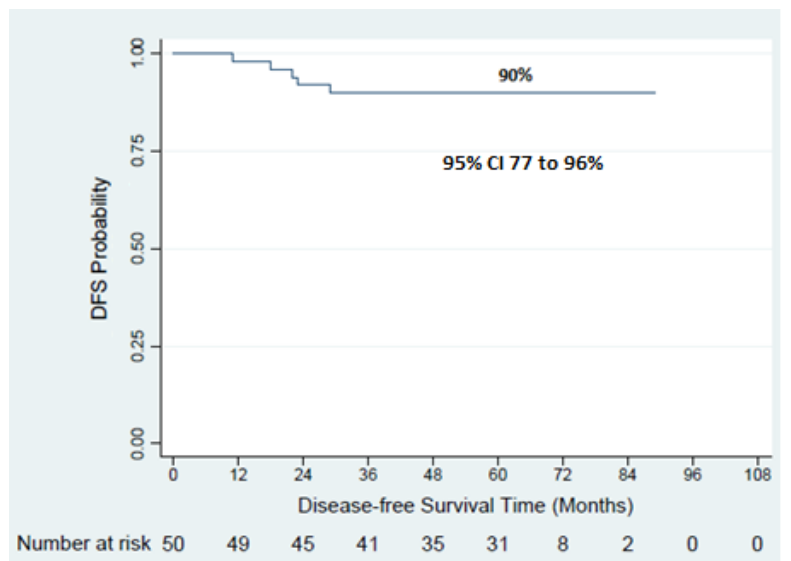

Figure 1.5-years disease free survival

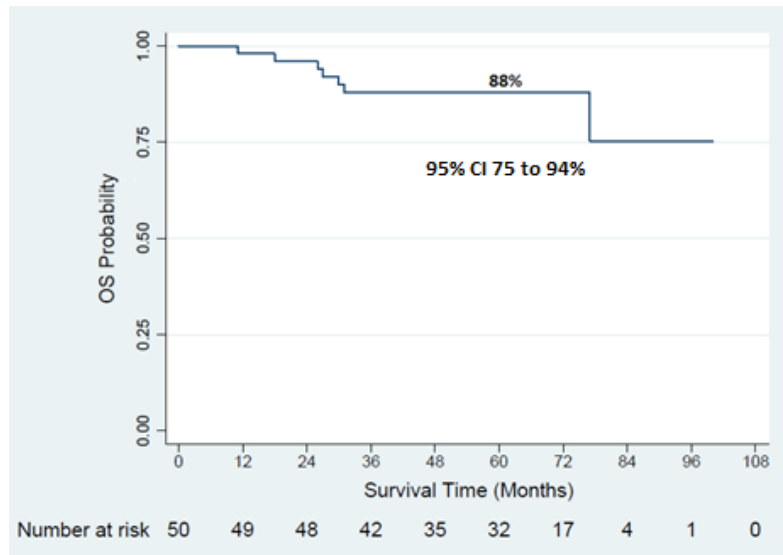

Figure 2.5-years overall survival

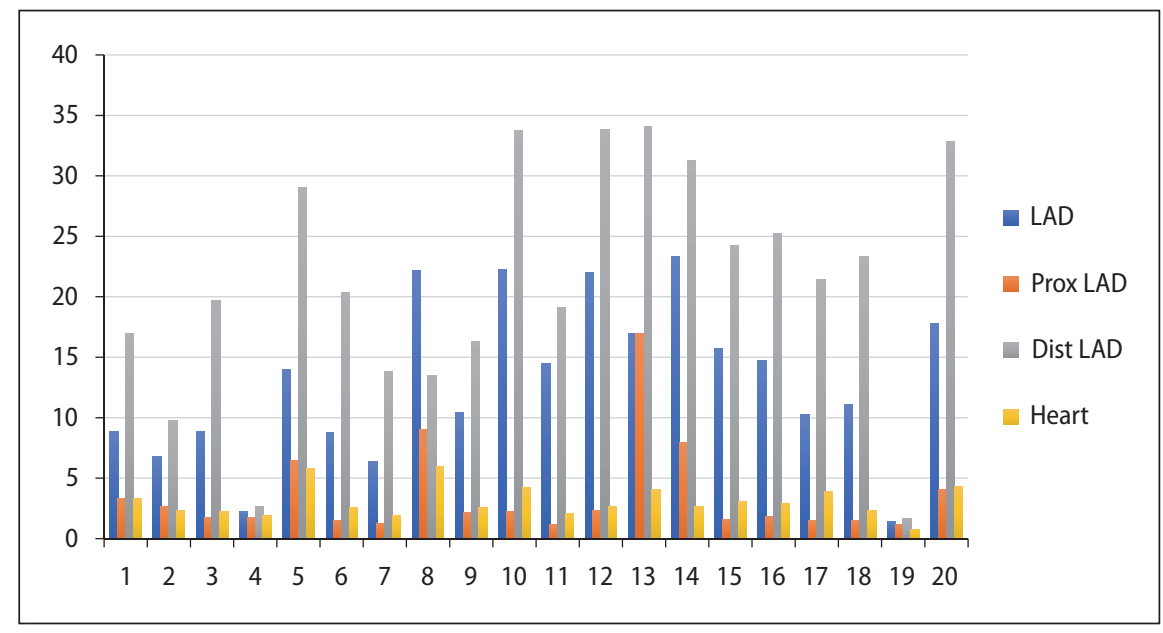

Figure 3. Mean doses to organs at risk (dose along Y-axis and patients along X-axis). LAD — left anterior descending coronary artery; prox — proximal; dist — distal

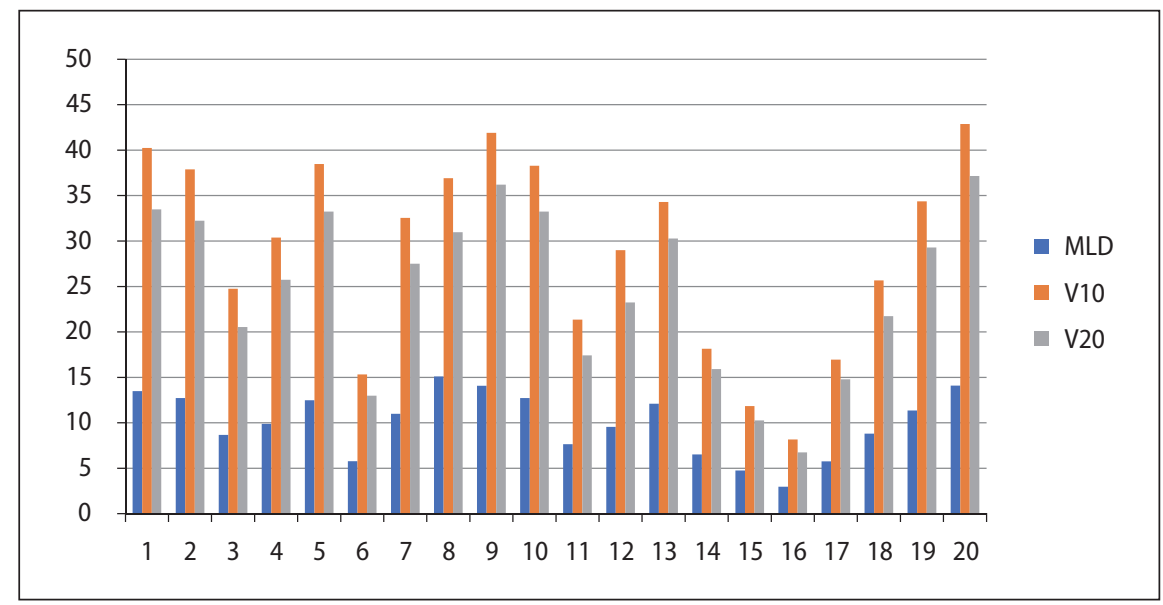

Figure 4. Left lung mean dose, V10 and V20 (dose along Y-axis and patients along X-axis). MLD — mean lung dose; V10 - volume receiving $10 \mathrm{~Gy}$; V20 — volume receiving $20 \mathrm{~Gy}$ 


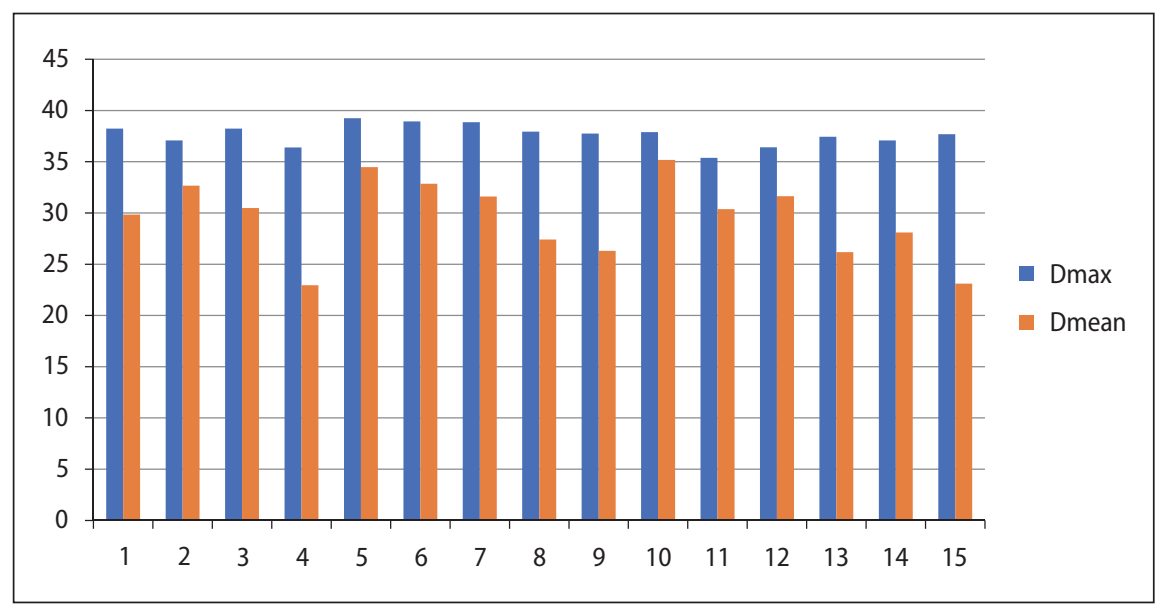

Figure 5. Brachial plexus Dmean and Dmax (dose along Y-axis and patients along X-axis)

with minimal toxicity and good cosmetic outcome [2]. With this 2 week schedule we could achieve all these objectives. Even after randomised trials from Canada [5] and the UK [3, 4, 7], optimal schedule with hypofractionation for post-operative breast cancer radiotherapy has not been defined yet. The START trials have concluded it to be 40 Gy in 15 fractions over 3 weeks for patients with early breast cancer. Studies are ongoing to further reduce the total dose as well as duration of treatment to 2 weeks or 1 week in early breast cancer. All this is possible because in the breast $\alpha / \beta$ of the tumor is similar to the $\alpha / \beta$ of the normal tissue. $\alpha / \beta$ for the local recurrence is $3.5 \mathrm{~Gy}$ and 3.1 Gy for the adverse effect [3, 4]. So, a high dose per fraction would be more effective on the tumor without much adverse effect on the normal breast tissue. In the present study, all late effects were within 2-6\%. These are well within the range reported in the randomized trials [3-7]. The START A trial reported that $38.2 \%$ of the breasts were indurated after hypofractionation as compared to $42.3 \%$ with conventional fractionation [3]. In our study, grade 2 breast induration was observed in only 1 (11\%) patient (Fig. 6). There was no grade 3 induration. Our results are also comparable to those reported by Wang et al. [8]. The RMH/GOC study reported that any difference in the breast appearance was $39.6 \%, 30.3 \%$ and $45.1 \%$ with $50 \mathrm{~Gy}$ in conventional fractionation, 39 Gy and $42.9 \mathrm{~Gy}$ in 13 fractions, respectively [7]. In the START trials, breast appearance change was $42.6 \%$ and $44.6 \%$ with conventional and hypofractionation, respectively. In our study, in patients with BCS, adverse effect on the breast was observed only in 2 (22\%) patients.

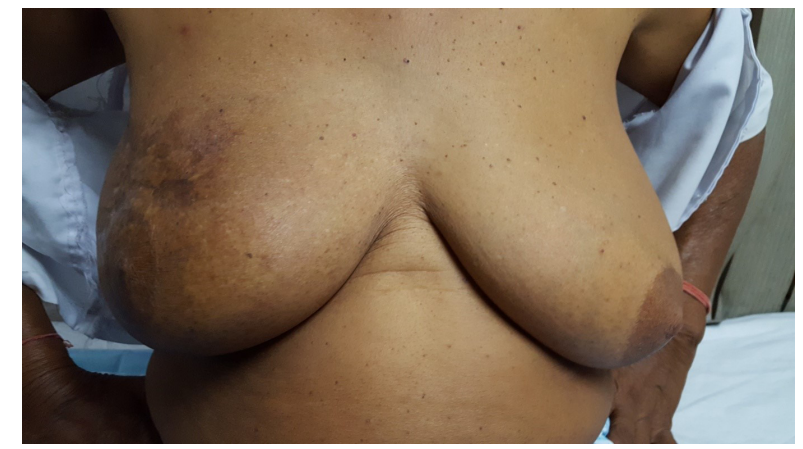

Figure 6. Late grade 2 breast induration and subcutaneous fibrosis

A Canadian study reported similar outcomes with hypofractionation and conventional fractionation [5]. They also reported that grade 2 subcutaneous toxicity increased three times over 10 years from $3.8 \%$ to $9.4 \%$ with hypofractionation as compared to $30 \%$ increase from $5.2 \%$ to $6.8 \%$ with conventional fractionation [6]. However, in the present study, the skin as well as subcutaneous toxicities did not deteriorate over time, but chest wall edema regressed with time. Subcutaneous toxicities at 3 and 5 -years were only $2 \%$ after postmastectomy radiotherapy (PMRT). In patients with BCS, subcutaneous toxicities at 3 and 5 years were $10 \%$ and $11 \%$, respectively. The increase in subcutaneous toxicities in the Canadian trial may be because of a higher total dose of $42.6 \mathrm{~Gy}$. The biological effective dose (BED) with this schedule would be $55.88 \mathrm{~Gy}$ and 80 Gy for early and late effects, respectively. The BED of $34 \mathrm{~Gy} / 10$ fractions/2 weeks would be $45.56 \mathrm{~Gy}_{10}$ and $72.53 \mathrm{~Gy}_{3}$ for early and late effects, respectively, in the case of PMRT. In the case of BCS with ad- 


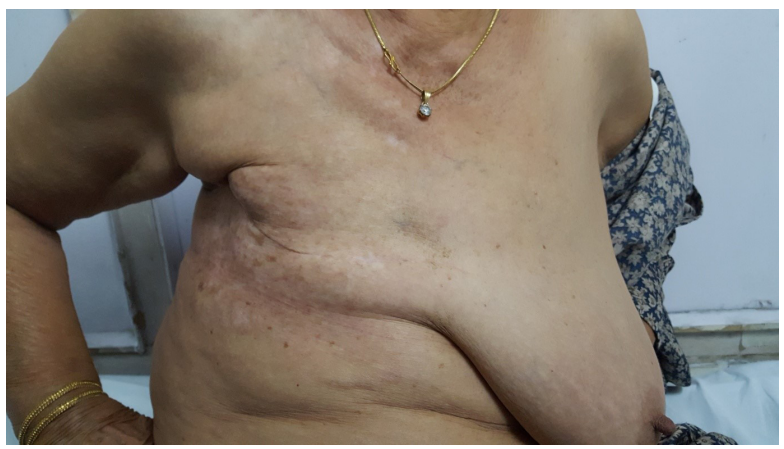

Figure 7. Late grade 2 hypopigmentation

ditional boost of $10 \mathrm{~Gy} / 5$ fractions/ 1 week to the tumor bed, the BED would be 57.56 Gy and 89.2 Gy, respectively. With hypofractionation, a small increase in the total dose can lead to significant differences in late toxicities. It was also observed in the START pilot trial where patients in the $42.9 \mathrm{~Gy}$ schedule had significantly increased breast induration; ( $40.8 \%$ vs. $20.4 \%$ vs. $28.6 \%$ with 42.9 Gy (3.3 Gy $\times 13$ fractions); 39 Gy (3 Gy in 13 fractions) and conventional fractionation, respectively [7]. Similarly, patients treated with 42.9 Gy also had significantly higher shoulder stiffness as compared to other START trials [10]. Recently, FAST forward trial also reported increased normal tissue adverse effects with $27 \mathrm{~Gy}$ as compared to $26 \mathrm{~Gy}$ [11].

Late grade 2 hypopigmentation was observed in $3(6 \%)$ patients at 5 years (Fig. 7).

Arm and shoulder functions were minimally affected with this schedule. Arm pain was reported by only $2(4 \%)$ patients, which is similar to $3 \%$ reported by Wang et al. [8] There was no deterioration in the arm and shoulder functions over time (Tab. 3). Arm/shoulder function impact rates are comparable to the START trials few patients who received RNI [10].

Moderate/marked shoulder/arm pain was reported in $8.6-12.1 \%$ and $11.4 \%$ patients in the START A and B trials, respectively. There was no RNI in the Canadian study [6].

In the present study, grade $\geq 2$ arm edema was seen in only 1 (2\%) patient (Fig. 8). In the START $\mathrm{A}$ and $\mathrm{B}$ trials, moderate/marked arm edema was reported in $3.3-4.8 \%$ and $3.5 \%$ patients, respectively. In another study, RNI with hypofractionation was not reported to add to arm edema [9]. In the current study, grade 2 shoulder stiffness was observed in only $2(4 \%)$ patients. Moderate/marked shoul-

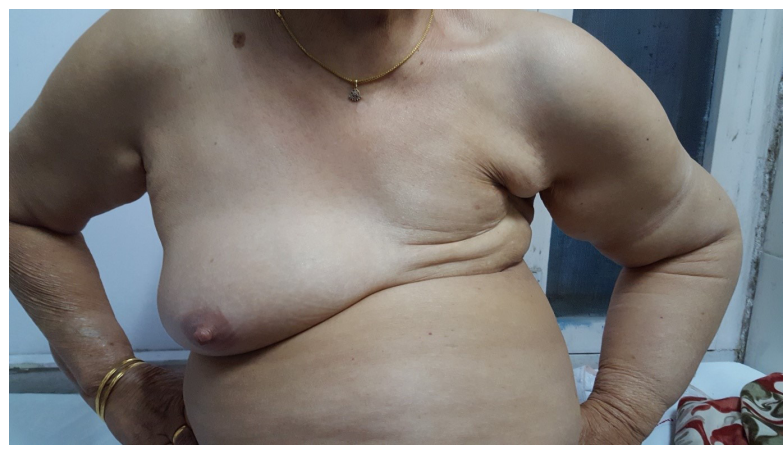

Figure 8. Late grade 2 arm edema (left)

der stiffness was reported in $3.4-6.9 \%$ and $5.6 \%$ patients in the START A and B trials, respectively [10]. Our results are also comparable to the study by Wang et al. where they reported lymphedema in $19 \%$ patients [8]. So, there was no detrimental effect of this 2-week schedule on arm/shoulder functions. There was no brachial plexopathy with this schedule as doses received were well within its tolerance limit. However, 1 patient in the START A trial treated with 41.6 Gy in 13 fractions was reported to have brachial plexopathy [10]. Based on the above studies, it appears that a dose of 34 Gy to 40 Gy delivered in 10 to 15 fractions may be safe for RNI.

5 years may be too short to report cardiac complications, but there were no cardiac or pulmonary toxicities with this schedule. It may be because the doses to the OARs were far lower than those reported with conventional fractionation. Only mean dose to the heart may not be relevant as it is quite low as compared to the dose to the distal $\mathrm{LAD}$, which may be responsible for coronary events (Fig. 6). So these patients need to be monitored for cardiac toxicity in the coming years although hypofractionation may be gentler to the heart because of low total nominal dose. Doses to the lung were also, within acceptable limits, so we did not encounter any pulmonary toxicity (Fig. 4). Recently, we published our long-term results of 3-week hypofractionation; moderate to marked arm pain, shoulder stiffness, late cardiac and pulmonary toxicities were observed in 254 (14.3\%), 219 (12.3\%), 29 (1.6\%) and $23(1.3 \%)$ patients, respectively [12].

So far, the local control with this schedule has been $100 \%$. Seven (14\%) patients have died, 5 $(10 \%)$ because of metastatic disease and $2(4 \%)$ because of infection. One patient died because of contra-lateral arm sepsis and another from lung 
infection. Since all dead patients were included for 5 -year OS calculation, it was $88 \%$ as compared to $90 \%$ DFS. These survival outcomes are also comparable to those reported in the randomised trials [3-8]. Wang et al. also reported $84 \%$ OS at 5 years in the hypofractionated radiotherapy group in their study of high risk patients [8].

Limitations of the study are the small cohorts of patients treated with a $2 \mathrm{D}$ technique, which may be of less relevance to institutes using 3D planning and treatment. There were no patients in this study with skin-sparing mastectomy or immediate reconstruction. Considering the safety and efficacy of this schedule, it is worth reporting it. Such hypofractionation schedule may help the radiation centers worldwide to reduce waiting time for radiotherapy, particularly in low-income countries where resources are limited. This schedule would also be of relevance in pandemic like covid-19 as it is economic, less time consuming, simple to execute and possible in any center in the world. It will also of economic importance to patients as they can complete their treatment fast and cheaper. Based on these encouraging outcomes, we have started a randomized trial to compare a 2-week with 3-week adjuvant loco-regional radiotherapy in patients with breast cancer for which enrollment will be completed by August 2020 (NCT04075058).

\section{Conclusion}

Hypofractionated radiotherapy in 2 weeks with this $2 \mathrm{D}$ technique in patients with breast cancer was associated with minimal late toxicity, good cosmetic outcome and excellent local control.

\section{Conflict of interest}

None declared.

\section{Funding}

SERB, India.

\section{References}

1. Smith BD, Bellon JR, Blitzblau R, et al. Radiation therapy for the whole breast: Executive summary of an American Society for Radiation Oncology (ASTRO) evidence-based guideline. Pract Radiat Oncol. 2018; 8(3): 145-152, doi: 10.1016/j.prro.2018.01.012, indexed in Pubmed: 29545124.
2. Bentzen SM, Agrawal RK, Aird EGA. START Trialists' Group,. The UK Standardisation of Breast Radiotherapy (START) Trial A of radiotherapy hypofractionation for treatment of early breast cancer: a randomised trial. Lancet. 2008; 9(4): 331-341, doi: 10.1016/S1470-2045(08)70077-9, indexed in Pubmed: 18356109.

3. Bentzen SM, Agrawal RK, Aird EGA. START Trialists' Group. The UK Standardisation of Breast Radiotherapy (START) Trial B of radiotherapy hypofractionation for treatment of early breast cancer: a randomised trial. Lancet. 2008; 371(9618): 1098-1107, doi: 10.1016/s01406736(08)60348-7, indexed in Pubmed: 18355913.

4. Whelan T, MacKenzie R, Julian J, et al. Randomized trial of breast irradiation schedules after lumpectomy for women with lymph node-negative breast cancer. J Natl Cancer Inst. 2002; 94(15): 1143-1150, doi: 10.1093/ jnci/94.15.1143, indexed in Pubmed: 12165639.

5. Yadav BS, Sharma SC. A Phase 2 Study of 2 Weeks of Adjuvant Whole Breast/Chest Wall and/or Regional Nodal Radiation Therapy for Patients With Breast Cancer. Int J Radiat Oncol Biol Phys. 2018; 100(4): 874-881, doi: 10.1016/j. ijrobp.2017.12.015, indexed in Pubmed: 29485066..

6. Whelan TJ, Pignol JP, Levine MN, et al. Long-term results of hypofractionated radiation therapy for breast cancer. N Engl J Med. 2010; 362(6): 513-520, doi: 10.1056/NEJMoa0906260, indexed in Pubmed: 20147717.

7. Yarnold J, Ashton A, Bliss J, et al. Fractionation sensitivity and dose response of late adverse effects in the breast after radiotherapy for early breast cancer: long-term results of a randomised trial. Radiother Oncol. 2005; 75(1): 9-17, doi: 10.1016/j.radonc.2005.01.005, indexed in Pubmed: 15878095.

8. Wang $\mathrm{SL}$, Fang $\mathrm{H}$, Song $\mathrm{YW}$, et al. Hypofractionated versus conventional fractionated postmastectomy radiotherapy for patients with high-risk breast cancer: a randomised, non-inferiority, open-label, phase 3 trial. Lancet Oncol. 2019; 20(3): 352-360, doi: 10.1016/s1470-2045(18)308131, indexed in Pubmed: 30711522.

9. Leong N, Truong PT, Tankel K, et al. Hypofractionated Nodal Radiation Therapy for Breast Cancer Was Not Associated With Increased Patient-Reported Arm or Brachial Plexopathy Symptoms. Int J Radiat Oncol Biol Phys. 2017; 99(5): 1166-1172, doi: 10.1016/j.jjrobp.2017.07.043, indexed in Pubmed: 29165285.

10. Haviland JS, Mannino M, Griffin C, et al. START Trialists' Group. Late normal tissue effects in the arm and shoulder following lymphatic radiotherapy: Results from the UK START (Standardisation of Breast Radiotherapy) trials. Radiother Oncol. 2018; 126(1): 155-162, doi: 10.1016/j. radonc.2017.10.033, indexed in Pubmed: 29153463.

11. Brunt A, Haviland J, Wheatley D, et al. FAST-Forward Phase III Randomised Controlled Trial of 1-Week Hypofractionated Breast Radiotherapy: 5-Year Results for Efficacy and Late Normal Tissue Effects. SSRN Electronic J. , doi: 10.2139/ssrn.3564389.

12. Yadav BS, Bansal A, Kuttikat PG, et al. Late-term effects of hypofractionated chest wall and regional nodal radiotherapy with two-dimensional technique in patients with breast cancer. Radiat Oncol J. 2020; 38(2): 109-118, doi: 10.3857/roj.2020.00129, indexed in Pubmed: 33012154. 